The fact that $\chi / \chi_{0}$ is less than 1 can be immediately interpreted as due to a penetration of the magnetic field into the particles, to a depth comparable to the particle size, while the fact that $\chi / \chi_{0}$ decreases with increasing temperature must mean that this penetration increases. It will be seen that $h / H_{c}$ increases just in the region where $\chi / \chi_{0}$ is decreasing, thus confirming the inference of Appleyard, Bristow and London $^{1}$ that this increase is associated with an increasing penetration depth. Similar results were obtained with an emulsion of mercury in chalk, but the effects were less pronounced owing to the larger average particle size.

Measurements were also made with a colloid of mercury in albumen in which the particle size was about ten times smaller than for the gelatine colloid, and the values of $\chi / \chi_{0}$ were now extremely small (Fig. 2), showing that the penetration of the field was almost complete. The critical fields, however, were so large that they could not be reached with the largest field used (900 gauss) even at the highest temperature.

If the law of falling-off of magnetic field into the surface of a superconductor is known, the value of $\chi / \chi_{0}$ can be calculated as a function of $r / \lambda$, where $r$ is radius of the sphere, and $\lambda$ the penetration depth. The London theory ${ }^{4}$ assumes the law to be according to the differential equation $\nabla^{2} H=H / \lambda^{2}$, and with this assumption it has been shown ${ }^{5}$ that

$$
\chi / \chi_{0}=1-3\left(\frac{\lambda}{r}\right) \operatorname{coth} \frac{r}{\lambda}+3 \frac{\lambda^{2}}{r^{2}},
$$

which for $r \ll 1$ reduces to

$$
\chi / \chi_{0}=\frac{1}{15} r^{2} / \lambda^{2} .
$$

Assuming this formula, we have calculated $\lambda / \lambda_{2} .5$ as a function of temperature for the albumen colloid, and as can be seen from Fig. 2 of Appleyard, Bristow and London's note, the results agree quite well with those from the thin mercury films, suggesting that equation (2) is of the correct form. At present only the order of magnitude of the average value of $r^{2}$ is known, so the absolute value of $\lambda_{2 \cdot 5}$ cannot be given exactly; it agrees, however, in order of magnitude with the thin film estimate. It will be noticed that close to the transition point, $T_{0}, \chi / \chi_{0}$ decreases linearly with $T_{0}-T$, which means that the penetration depth becomes infinite at $\left(T_{0}-T\right)^{-1 / 2}$.

In the case of the larger particles, as in the gelatine colloid and the chalk emulsion, the London assumption cannot be tested exactly, since the lack of uniformity of particle size complicates the application of equation (1); this complication does not arise in the case of particles so small that equation (2) can be used. It is hoped to overcome this difficulty by working with a more uniform preparation of known particle size.

In conclusion, it is a pleasure to thank Mr. R. T. M. Haines, of British Colloids, Ltd., for preparing the colloids used in these experiments.

Royal Society Mond Laboratory,

$$
\text { D. SHOENBERG. }
$$

Cambridge.

Fob. 6.

\footnotetext{
1 Appleyard, Bristow and London, see letter above.

2 Pontius, Phil. Mag., 24, 787 (1937).

3 Shalnikov, NATURE, 142, 74 (1938); Appleyard and Misener, NATURE, 142, 474 (1938).

‘ London, F. and H., Proc. Roy. Soc., A, 149, 71 (1935). 'London, F., Physica, 3, 450 (1936). von Laue, Ann. Phys., 32,
}

\section{Atomic Nuclei in Primary Cosmic Radiation}

IT is generally assumed that the mesotrons in cosmic radiation are produced by primary electrons in the upper atmosphere, but there seems as yet not to be any direct evidence for such a process. On the contrary, experiments by Jánossy ${ }^{1}$ indicate that electrons cannot produce mesotrons with a prob. ability sufficient to account for the intensity of the mesotronic component.

According to a recent theory of the origin of the cosmic rays ${ }^{2}$, these are likely to constitute a more or less fair sample of the charged particles in the neigh. bourhood of the stars (especially the double stars). This means that the primary cosmic radiation is likely to contain electrons but also a considerable fraction of atomic nuclei with very high energies.

The number of protons or other nuclei observed at sea-level is, however, very small. This might be accounted for by the assumption that incident highenergy nuclei are strongly absorbed when colliding with nuclei in the atmosphere. Such an absorption could be due to a nuclear correspondence to the Bremsstrahlung ${ }^{3}$, which would imply that it is accompanied by the emission of mesotrons. This would explain the origin of the mesotrons in cosmic radiation at the same time. Processes of this kind must be accompanied by nuclear explosions of the kind observed especially at high altitudes. As the frequency of the explosions increases extremely rapidly with the altitude, it is improbable that they are caused by the mesotronic or electronic components.

In order to fit observations, the nuclear absorption cross-sections in air must be at least $\sim 2 \times 10^{-25} \mathrm{~cm} .^{2}$ which at the energies concerned corresponds to $1.4 \times 10^{-26} \mathrm{~cm} .^{2}$ in hydrogen. For the latter, Euler and Heisenberg ${ }^{8}$ give as a preliminary theoretical value $\sim 10^{-26} \mathrm{~cm}^{2}$.

Hannes Alfvén.

Forskningsinstitutet för Fysik, Stockholm 50 .

Feb. 6.

${ }_{1}$ Jánossy, L., Proc. Camb. Phil. Soc., 34, 614 (1938).

${ }^{2}$ Alfvén, H., Z. Phys., 107, 579 (1937); Compare Phys. Rev., 54, 97 (1938).

${ }^{3}$ Euler, H., and Heisenberg, W., Erg. d. ex. Naturw., 17, 30 (1938).

\section{Bursting of Glass Tubes by Thixotropic Pastes}

Iv a test for the liquid absorption of light powders, water is gradually added to the powder in a hardglass thick-walled test-tube fitted with a rubber bung. This is shaken between additions by giving several successive brisk taps each with the closed and the stoppered ends downwards on to a rubber pad until a paste is produced which flows out under certain standard conditions. The tubes, of $155 \mathrm{~mm}$. length and $22 \mathrm{~mm}$. bore, are about half full at the com. pletion of the test.

Occasionally, while tapping the tube stopper downwards, the end of the tube bursts outwards and the main bulk of the paste is violently thrown a considerable distance as a single blob. The pastes are strongly thixotropic (false-bodied) and the bursting occurs at the stage when the paste appears quite fluid during shaking but sets rapidly and will not flow out. On these occasions the operator is not sensible of having used excessive force; indeed, 